\title{
A NOTE ON INFLUENCE OF SUBGROUP RESTRICTIONS IN FINITE GROUP STRUCTURE
}

\author{
R. KHAZAL \\ Department of Mathematics \\ Kuwait University \\ P.0. BOX 5969 - 13060 - Kuwait

\section{N.P. MUKHERJEE} \\ Jawaharlal Nehru University \\ New Delhi - 110067, Ind I a \\ (Received Apri1 25, 1988 and in revised form July 7, 1988)
}

ABSTRACT. The structure of a finite group under specific restrictions respectively on its maximal, minimal and prime power subgroups has been investigated in this paper.

KEY WORDS AND PHRASES. Maximal subgroup, minimal subgroup, Frobenius Kernel, Supersolvable, nilpotent. 1980 AMS SUBJECT CLASSIFICATION CODE. Primary 20E28, Secondary 20 D10.

1. INTRODUCTION.

We consider in this note group theoretic restrictions on specific subgroups of a finite group G. These restrictions yield different characterizations of G. All the groups considered in this note are finite. We shall use a result due to $R$. Baer [1, Lemma 3, pp. 12] in proving Theorem 1 and we state it below for the sake of completeness.

LEMMA 1. If the group $G$ possesses a maximal subgroup with core 1 then the following properties of $G$ are equivalent.

(1) The indices in $G$ of all the maximal subgroups with core 1 are powers of one and the same prime p.

(2) There exists one and only one minimal normal subgroup of $G$, and there exists a common prime divisor of all the indices in $G$ of all the maximal subgroups with core 1 .

(3) There exists a soluble norma1 subgroup, not 1 , in $G$. 


\section{GENERAL RESULTS.}

THEOREM 1. If the indices of all non normal maxinal subgroups of a group $G$ are equal then $G$ is solvable.

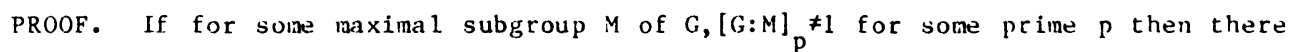
exists however a maximal subgroup $M^{*}$ such that $\left[G: M^{*}\right]_{p}=1$. Therefore $G$ is not simple and by induction $G / V$ is solvable and $V$ is unique. If $N \subseteq \phi(G)$ then $G=x N$ for some maximal subgroup $X$ of $G$ and $X$ is core free. Since the indices of all core free maxinal subgroups are same it now follows by Lema 1 that $\mathrm{N}$ is solvable which implies G is solvable.

PROPOSITION 1 . If a non abelian group $G$ has a maximal subgroup $M$ whose intersection with any other maximal subgroup is trivial then $G$ must be elementary abelian by cyclic, $G$ is an SBP (Sylow best possible) group and matches the description of Theorem 2.2 [5].

PROOF. If $M$ is the only maxinal subgroup of $G$ then $G$ is cyclic and also note that $G$ cannot be a p-yroup either. For if $M_{1}$ and $M$ are two maximal subgroups of $G$ thẹn each one of them is normal in $G$ and $\left|M_{1}\right|=|M|=p^{n-1}$ if $|G|=p^{n}$. This implies $|G|=p^{n}=\left|M_{1}\right| \cdot|M|=p^{2 n-2}$ i.e. $N=2$ and $G$ is abelian. Now $M \cap M^{x}=\langle e\rangle \forall x$ in $G$ implies $G=F M, F \cap M=\langle e\rangle$ where $F$ is the Frobenius Kernel. Consequently $F$ is elementary abelian for some prime $p$. If $M_{0}$ is a maximal subgroup of $M$ then $F M_{0}$ is also a maximal subgroup of $G$ and hence $G=F\langle x\rangle,|\langle x\rangle|=q=a$ prime.

The location of the prime ordered elements and elements of order 4 in the center of a group $G$ imply that $G$ is nilpotent. This theorem is due to N. Ito (Theorem 5.5, pp. 435 [2]). The following theorem is a dual of Ito's result.

THEOREM 2. A non abelian group $G$ in which every minimal subgroup is self centralizing is a group of order $p^{\alpha} q, p, q$ are different primes.

PROOF. Let $x$ be an element of $G$ such that $|x|=p_{n}$, the smallest prime divisor of $|G|$. If an element $y$ normalizes $\alpha_{i}\langle x\rangle$ then $y_{i}, \forall_{i}$ also normalizes $\langle x\rangle$ where $\langle y\rangle$ $=\left\langle y_{1}\right\rangle \times\left\langle y_{2}\right\rangle \times \ldots \times\left\langle y_{n}\right\rangle,\left|y_{i}\right|=p_{i}, p_{1}=a$ prime. Therefore $\left\langle y_{i}\right\rangle\langle x\rangle=\langle x\rangle\left\langle y_{1}\right\rangle$ is supersolvable and so $\mathrm{Y}_{i} \mathrm{~V}_{\mathrm{i}}$ centralizes $\mathrm{x}, \mathrm{l}<\mathrm{i}\left\langle\mathrm{n}=1\right.$. In $\left\langle\mathrm{y}_{\mathrm{n}}\right\rangle\langle\mathrm{x}\rangle=\langle\mathrm{x}\rangle\left\langle\mathrm{y}_{\mathrm{n}}\right\rangle=\mathrm{H}$, $\left\langle y_{n}\right\rangle$ is a maximal subgroup and consequently is normal in $H$. (If $\langle x\rangle \subseteq\left\langle y_{n}\right\rangle$ then of course $y_{n}$ trivially centralizes $x$ ). It follows that $y_{n}$ and $x$ centralize each other and we therefore conclude $\langle x\rangle \cap\langle x\rangle^{g}=\langle e\rangle \nabla_{g}$ in $G \backslash\langle x\rangle$. Thus $G=F\langle x\rangle, F \cap\langle x\rangle=\langle e\rangle$, $F$ is the Frobenius Kernel. Evidently $|F|$ is divisible by one prime and $F=\langle y\rangle,|\langle y\rangle|$ = a prime power.

PROPOSITION 2. If the order of a group $G$ is divisible by at least two primes and every proper subgroup is of prime power order then $G$ is elementary abelian by cyclic, $G$ is an SBP (Sylow best possible) group and matches the description of Theorem 2.2 [5]. 
PROOF. Evidently every Sylow p-subgroup of $G$ is a maximal subgroup and therefore $G$ is solvable [6]. If $N$ is a minimal normal subgroup of $G$ then $G=N Q,(|N|,|Q|)=$ 1. This however implies $G=N\langle x\rangle,|x|=q$ for some prime $q$.

The motivation for the next theorem is derived from the fact that Sylow subgroups corresponding to same prime in a subgroup $H$ of a group $G$ are conjugate in $H$ itself. It characterizes groups in which not only sylow subgroups but prime power subgroups of same order are conjugate in each subgroup.

THEOREM 3. If subgroups of same prime power order are conjugate in any subgroup of $G$ then $G$ is supersolvable, the Sylow p-subgroups for $p>2$ are cyclic and the Sylow 2-subgroup has a cyclic normal subgroup of index 2 .

PROOF. By induction every maximal subgroup of $G$ is supersolvable and so $G$ is solvable. Let $\mathrm{N}$ be a minimal normal subgroup of $\mathrm{G}$. $\mathrm{G} / \mathrm{N}$ is supersolvable by induction and since $\mathrm{N}$ is elementary abelian it follows that $|\mathrm{N}|=\mathrm{p}$, a prime. Therefore $\mathrm{G}$ is supersolvable.

Now suppose $X$ is a minimal normal subgroup of $G$ and let $|x|=p$. Note that $G$ has

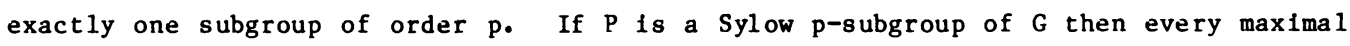
subgroup of $P$ has exactly one subgroup of order $p$ and by induction is cyclic. Therefore all abelian normal subgroups of $P$ are cyc11c. By Theorem 7.6 [2, p. 304] $P$ is cyclic if $p>2$. If $p=2$ then $P$ has a cyclic normal subgroup of index 2 .

Consider $G / X$ and by induction all its Sylow p-subgroups for $p>2$ are cyclic and a Sylow 2-subgroup has a cyclic maximal subgroup of index 2. This however implies that the Sylow subgroups of $G$ have the desired property and the theorem is proved completely.

THEOREM 4. If every minimal subgroup of a group $G$ is complemented in $G$ then $G$ is supersolvable.

PROOF. Let $H$ be any subgroup of $G$ and $\langle a\rangle$ be a minimal subgroup in $H$. Then $G=$ $\langle a\rangle T,\langle a\rangle \cap T=\langle e\rangle$. Consequently, $H=\langle a\rangle(H \cap T),\langle a\rangle \cap(H \cap T)=\langle e\rangle$. By induction it now follows that every maximal subgroup of $G$ is supersolvable and therefore $G$ is solvable [5, Theorem 2.3, pp. 10]. Let $N$ be a minimal normal subgroup of $G$. If $b \in N$ then $G=\langle b\rangle K,\langle b\rangle \cap K=\langle e\rangle$ and $K$ is a maximal subgroup of $G$. This implies $N=\langle b\rangle$ ( $N \cap K$ ) by Dedekind's modular 1 aw and $N$ being minimal normal in $G$ it follows that $\mathrm{N} \cap \mathrm{K}=\langle e\rangle$. Hence $\mathrm{N}=\langle\mathrm{b}\rangle$ and $\mathrm{G} / \mathrm{N}$ are supersolvable which however imply that $\mathrm{G}$ is supersolvable.

REMARK. The Sylow subgroups of such a group $G$ as stated in the theorem are not necessarily cyclic as the instance of $S_{3}$ might suggest.

Let $G=\left\langle a, b, x \mid a^{3}=b^{3}=1, a b=b a, a^{x}=a^{2}, b^{x}=b^{2}, x^{2}=e\right\rangle . \quad G$ is a group of order 18 which is supersolvable and every minimal subgroup is complemented in $G$. However, the Sylow 3-subgroup of $\mathrm{G}$ is not cyclic. 


\section{REFERENCES}

1. BAER, R., Clisses of fintte groups anit their properties, Illinois J. Math. 1 (1957), 115-197.

2. HUPPERT, B. Eudlichen Gruppen I, Springer, N.Y. 1967.

3. MUKHER.JEE, N.P., The hyperquasicenter of a finite group II, Proc._Aner. Soc. 32 (1972), 24-28.

4. MUKHERJEE, N.P., A note on normal index and maxinal subgroups in finite groups, Illinois J. Math. 2 (1975), 173-178.

5. WE INSTEIN, M., Between Nilpotent and Solvable, Polygonal Publishing House, NJ, 1982.

6. THOMPSON, J., Proof of a conjecture of Frobenius, Proc. Symp. Pure Math. Amer. Math. Soc. 1 (1959). 


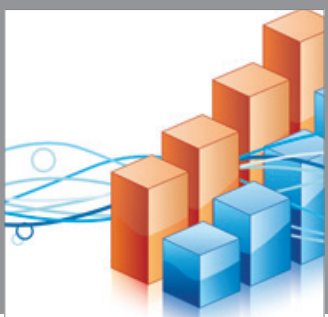

Advances in

Operations Research

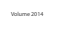

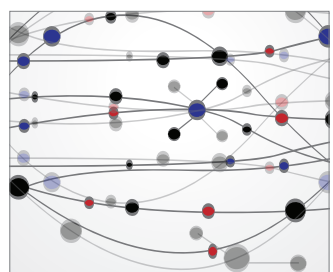

\section{The Scientific} World Journal
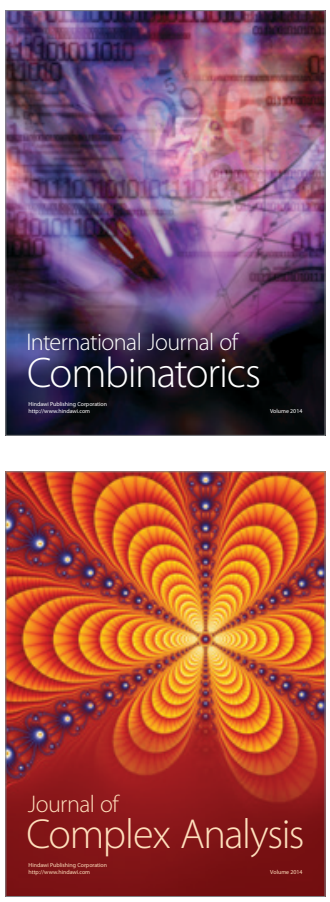

International Journal of

Mathematics and

Mathematical

Sciences
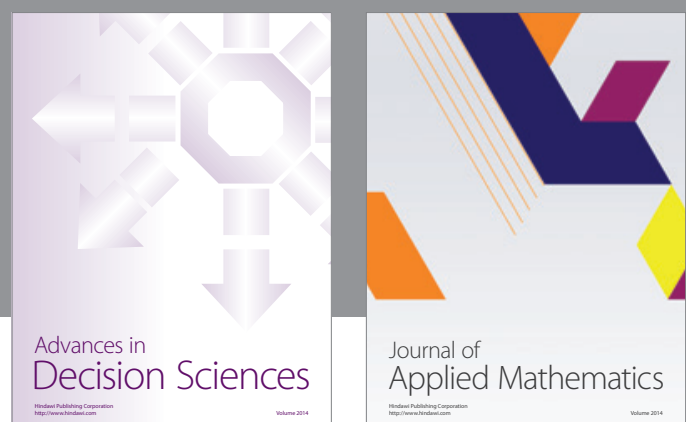

Journal of

Applied Mathematics
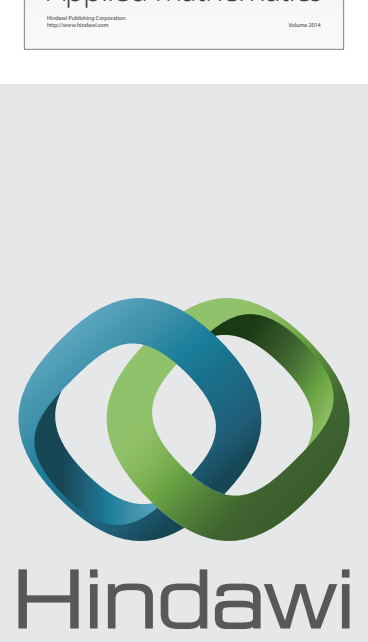

Submit your manuscripts at http://www.hindawi.com
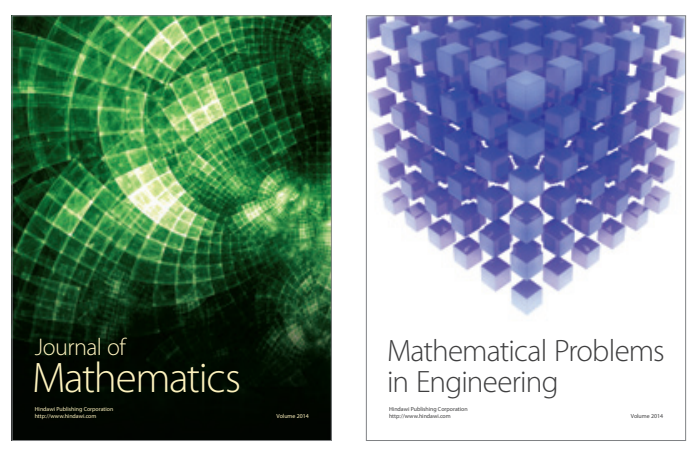

Mathematical Problems in Engineering
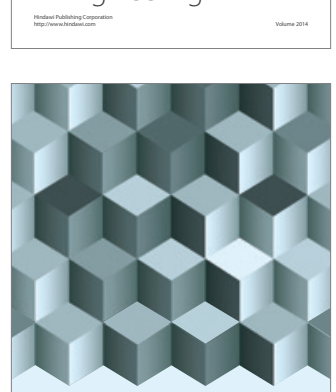

Journal of

Function Spaces
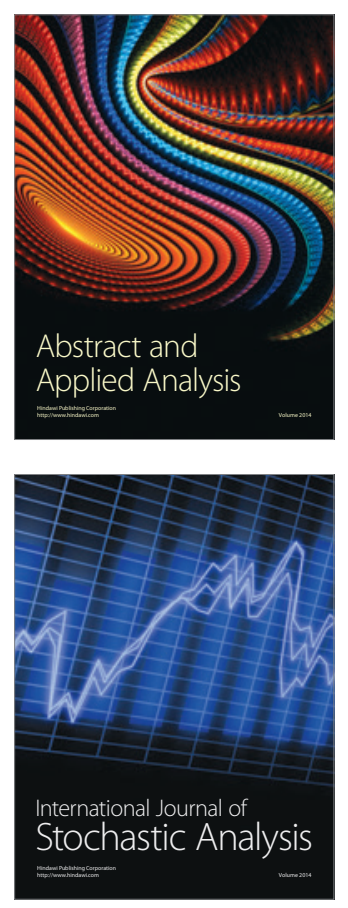

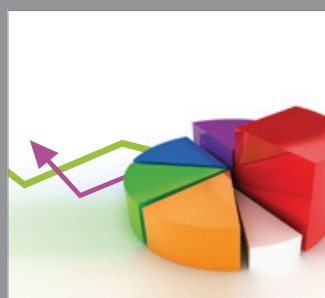

ournal of

Probability and Statistics

Promensencen
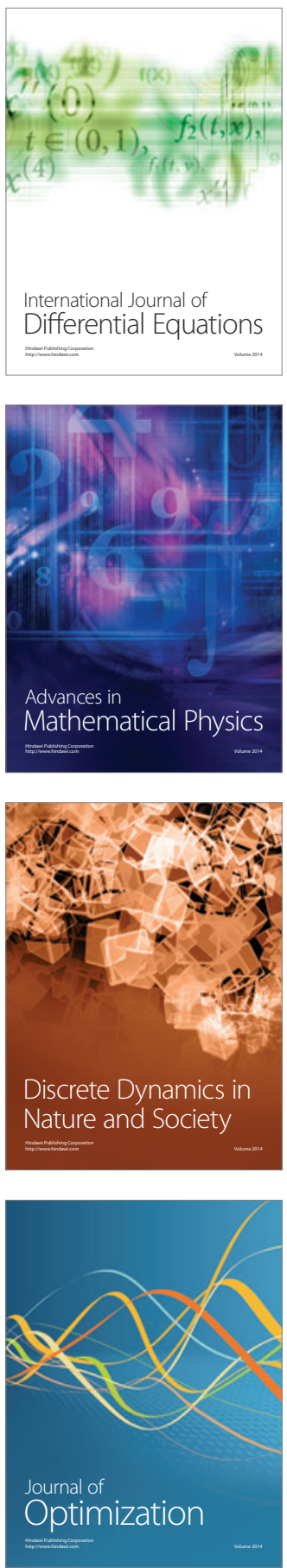